\title{
Los sistemas de Evaluación de la Educación Superior en México y España
}

\author{
García García, César \\ CIIDET/Tecnológico Nacional de México, Querétaro, México \\ cesargarcia1810@gmail.com \\ Hervás Torres, Mirian \\ Universidad de Granada, Granada, España \\ miriamhervas@ugr.es
}

\section{Resumen}

Desde la convergencia e integración mundial de la Educación Superior, la conformación de sus sistemas de evaluación está en el punto de mira, apreciándose el esfuerzo de los gobiernos para conducir, regular y vigilar a las Instituciones de Educación Superior. Esta evaluación se centra en las funciones sustantivas de docencia, investigación y vinculación, pero también las funciones adjetivas de gestión, bajo la lógica de la calidad, principios de eficiencia, eficacia, transparencia y rendición de cuentas a la sociedad. Mediante el método comparado se contrasta los sistemas de evaluación de Educación Superior entre México y España, obteniendo como resultados la similitud de programas gubernamentales enfocados en la evaluación de la docencia con estándares e indiciadores de actividades de investigación, y al mismo tiempo se aprecian diferencias en los marcos históricos de conformación de los sistemas de evaluación, los tipos de agencias centralizadas (caso de España) o descentralizadas (caso de México).

\section{Abstract}

From convergence and global integration of Higher Education, the establishment of evaluation systems is in the crosshairs, appreciating the efforts of Governments to lead, regulate and monitor the Institutions of Higher Education. This assessment focuses on the substantive functions of teaching, research, and connection, but also the procedural functions of management, under the logic of the quality, principles of efficiency, effectiveness, transparency and accountability to the society. Through comparative method it will be contrasts the evaluation systems of Higher Education between Mexico and Spain, obtaining as a result the similarity of governmental programs focused on the evaluation of teaching with standards and indicators of research, and at the same time there are differences in historical frameworks of formation of systems of evaluation, the types of centralized agencies (case of Spain) or decentralized (case of Mexico).

Palabras clave: Educación Superior, evaluación, acreditación, certificación, programas.

Keywords: Higher Education, assessment, accreditation, certification, programs.

\section{INTRODUCCIÓN}

Diversos autores (Schirewer, 1997; Altbach, 2001) coinciden que en el marco de la globalización hay una convergencia y una diferenciación de los sistemas de Educación Superior (ES) a nivel mundial. Por ello, es necesario comparar cómo son los sistemas de evaluación para comprender sus semejanzas, diferencias y aprendizajes que sirvan para el desarrollo de políticas educativas mejoradas.

Se entiende que los sistemas de evaluación son un esfuerzo de los gobiernos para conducir, regular y vigilar a las instituciones de ES. Esta evaluación se centra en las funciones sustantivas de docencia, investigación y vinculación, y en las funciones adjetivas de gestión, bajo los principios de calidad, eficiencia, eficacia, transparencia y rendición de cuentas a la sociedad. 
Con este marco, México y España han constituido sus sistemas de evaluación formales, permanentes y sistemáticos mediante un conjunto de instituciones, políticas, programas gubernamentales y procesos académicos, de gestión y organización, que necesitan ser revisados.

El presente trabajo mediante el método comparado toma como objeto de análisis los sistemas de evaluación en un marco histórico-político centrado en el interés de los estados, las instituciones y programas de evaluación, calidad, acreditación y certificación de la ES. Se plantea un contraste entre políticas, programas gubernamentales y procesos de evaluación, y se extraen las diferencias y semejanzas de los sistemas para entender las causas (Schriewer, 1998); comprender qué puede servir para un sistema educativo, qué no funciona y qué cambiar y ajustar (Cox, 2003); ahondar en la discusión, diseño e implementación de políticas para el sector educativo terciario; e incluir las perspectivas y las experiencias globales (Albatch, 2001).

\section{EL SISTEMA DE EVALUACIÓN DE LA EDUCACIÓN SUPERIOR EN MÉXICO}

En México la evaluación de la ES se produce en los años 70. Los primeros ejercicios estuvieron relacionados con procesos de planeación gubernamental con fines de diagnóstico, pronósticos de demanda y crecimiento, así como programas específicos de desarrollo institucional. En los años 80 la evaluación se empezó a asociar en la conducción de gobierno y de pensar las políticas para ES bajo principios de eficiencia y eficacia.

Sin embargo, la mayoría de los investigadores nacionales (Buendía, 2011; Mendoza, 2003; Rubio, 2007) coinciden en que propiamente el sistema nacional de evaluación comienza en los años 90 con la edificación de un conjunto de agencias de evaluación y programas gubernamentales de financiamiento extraordinario para conducir, regular y vigilar a las Instituciones de Educación Superior (IES).

\subsection{El periodo de modernización y evaluación}

En la década de los 90, conocida como periodo de modernización, la evaluación pasó de ser un instrumento de planeación universitaria a ser un mecanismo de regulación y conducción de las universidades (Mendoza, 2003), mediante valores como la búsqueda de calidad, del financiamiento extraordinario, la rendición de cuentas (accountability) y modelos de gestión empresarial (Buendía, 2011).

De acuerdo a Mendoza (2003) el sistema de evaluación de la ES se estableció a partir del Programa de Modernización Educativa (1989-1994), del cual se establecieron tres líneas de acción: (a) la evaluación que realizarían las propias instituciones (autoevaluación), (b) la evaluación del sistema y de los diversos subsistemas que lo conformaban por especialistas nacionales y extranjeros, y (c) la evaluación interinstitucional, mediante un esquema de evaluación por pares académicos calificados.

La autoevaluación que realizarían las propias universidades, se planteó con fines de diagnóstico y mejora, donde todas las universidades estatales fueron evaluadas al $100 \%$. En el caso de la evaluación del sistema por especialistas nacionales y extranjeros, se consideró la participación de la UNESCO y de la OCDE. Los resultados aparecieron en un documento de 1997 titulado, Exámenes de las Políticas Nacionales de Educación Superior.

Finalmente para la evaluación interinstitucional, se creó un sistema de evaluación por pares, la forma más legítima fue a través de los Comités Interinstitucionales para la Evaluación de la Educación Superior (CIEES), organismo no gubernamental integrado por pares académicos, quienes estaban en mejores condiciones dotados de opinar sobre la calidad de un programa académico que se ubicaba en su campo de conocimiento.

\subsection{Una nueva década de evaluación...y acreditación}

A finales del 2000 se continúa con la meta de conformar un sistema nacional de evaluación, para ello se introduce un mecanismo inédito, la acreditación. En este proceso, la evaluación se enten- 
día como una estrategia gubernamental que permitía detectar desigualdades de calidad en los programas educativos, para propiciar la mejora continua y el aseguramiento de la calidad de los mismos. Mientras la acreditación se identificó como un medio adecuado para lograr el reconocimiento social y el prestigio (Rubio, 2007, p.36).

En esta década, a solicitud de la Secretaría de Educación Pública (SEP), organismo gubernamental encargado de regular la educación del país, los CIEES empezaron a evaluar las IES tecnológicas y algunas IES particulares que lo solicitaran. Finalmente los CIEES, conformaron un padrón de programas educativos para ubicarlos en distintos niveles de calidad.

De la misma manera, la Federación de Instituciones Mexicanas Particulares de Educación Superior (FIMPES), organismo que defiende la presencia, interés y pertinencia de la educación privada en el sistema educativo nacional, logró en el año 2000 funciones de acreditación de sus asociados. En esta década poco más del $6 \%$ de las IES privadas contaban con reconocimiento de su calidad (es decir, estaban acreditadas) y era evidente la falta de regulación de la calidad de las universidades privadas (Mendoza, 2003). En la misma década, nace el Consejo para la Acreditación de la Educación Superior (COPAES), cuya función consiste en reconocer a las agencias de acreditación, las cuales son las encargadas de otorgar las acreditaciones a los programas educativos.

\subsection{Los programas gubernamentales de evaluación y acreditación}

La entrada para la evaluación de la ES fue su carácter no obligatorio y con fines de diagnóstico, hasta evolucionar en diversas instituciones y programas gubernamentales de evaluación y acreditación que comenzaron a evaluar la investigación y la docencia, asociados a la asignación de recursos económicos (el financiamiento extraordinario).

En este tenor, tres programas gubernamentales se pueden mencionar: el Sistema Nacional de Investigadores (SNI), el Programa del Mejoramiento del Profesorado (PROMEP), y el Programa Nacional de Posgrado de Calidad (PNPC).

EI SNI para evitar la fuga de cerebros frente a la crisis económica ofrecía un apoyo económico a los científicos e investigadores más sobresalientes de las IES públicas. Actualmente es un Programa que evalúa la calidad de los investigadores y aparece como indicador de evaluación de otros programas de mejora. Asimismo, es el Programa más longevo que se ha modernizado para evaluar a los investigadores, contando actualmente con 27 mil investigadores.

En el caso del PROMEP aplicó sólo para IES públicas con el propósito de mejorar la formación académica de los profesores universitarios e incrementar la figura de Profesores de Tiempo Completo (PTC) asociados a actividades de investigación.

Con la expansión de la ES muchos de los profesores sólo tenían estudios de pregrado (licenciatura), con el PROMEP se buscaba que tuvieran un grado de formación mayor al que impartían la enseñanza. Este objetivo prácticamente se logró con creces. Actualmente hay una política gubernamental donde para la contratación de profesores en las IES públicas el nivel académico de contratación que se requiere es de maestría o doctorado.

Finalmente el PNPC se crea para atender el problema de la masificación de los posgrados y mejorar su calidad, lo cual se busca a partir de conseguir mejorar determinados indicadores (plan de estudios, personal académico, estudiantes, infraestructura y vinculación). La acreditación no es permanente, sino se da por niveles y por temporalidad. Existen Programas de posgrado de reciente creación, en desarrollo, consolidados, y competencia internacional, los cuales son evaluados cada 3 años y pueden subir de nivel o salir del Programa.

Finalmente, el gobierno creó el Programa Integral de Fortalecimiento Institucional (PIFI), un instrumento de planeación y evaluación de mejora institucional que ha servido hasta el momento para la asignación de recursos extraordinarios a las IES públicas. 


\section{EL SISTEMA DE EVALUACIÓN DE LA EDUCACIÓN SUPERIOR EN ESPAÑA}

España como país joven en su sistema de evaluación se ha visto obligado al cambio ante un entorno de integración dentro del Espacio Europeo de Educación Superior (EEES).

En la Reforma Universitaria de 1983 su interés residía en la evaluación del rendimiento docente y científico del profesorado, posteriormente con la Ley Orgánica de Universidades del 2001 se orientó hacia la calidad de las IES con respecto a la rendición de cuentas, transparencia, cooperación y competitividad de las universidades, información a la administración y a la sociedad, mejora de la actividad docente, investigadora y de gestión de las universidades (Barrenetxea et al., 2005). Esto dio lugar a procesos de evaluación, certificación y acreditación.

En este proceso, España se percata de la necesidad de contar con sistemas de evaluación y acreditación de calidad compatibles y precisos para conseguir el reconocimiento mutuo entre las diferentes instituciones educativas europeas, y constatar la existencia de los niveles de calidad prefijados (Haug, 2003).

Esto se produce en un periodo de 15 años, con la armonización de los estudios universitarios oficiales españoles mediante las directrices establecidas en el Proceso de Bolonia (Agencia Nacional de Evaluación de la Calidad y Acreditación -ANECA-, 2016), llegando al acuerdo de Grados (4 años), Máster (1 año) y Doctorado (4 años), lo cual ha supuesto la edificación de un sistema de evaluación, fundando la ANECA, y posteriormente las agencias especializadas de evaluación por Comunidades Autónomas, que integran programas enfocados en la evaluación, acreditación y certificación.

\subsection{Los programas de Evaluación, Acreditación y Certificación}

La ANECA creada en 2001 con el propósito de integrar competitivamente las IES españolas al EEES, aportar garantía externa de calidad al sistema universitario mediante la evaluación, la certificación y la acreditación de enseñanzas, profesorado e instituciones (European Commission/ EACEA/Eurydice, 2015). Creó una serie de Programas que dan cuenta de la evaluación centrada hacia la docencia, en las enseñanzas y las instituciones.

Su labor con el docente se dirige a la creación de los programas PEP (Programa de Evaluación del Profesorado), ACADEMIA, DOCENCIA y CNEAI.

EI PEP creado para la contratación del docente universitario, bajo diferentes figuras (profesor ayudante de doctor, profesor contratado y profesor de universidad privada), evalúa su formación académica, formación más especializada (p.e., estancias posdoctorales), o experiencia docente e investigadora (publicaciones científicas en revistas de prestigio), entre otras. Según los datos recogidos su impacto arroja altos niveles de exigencia en la evaluación docente y la pretensión de esta agencia de conseguir personal altamente cualificado. No obstante, esta exigencia ha generado una teoría del embudo, donde los nuevos profesores tienen menos opciones de acreditarse y a la vez las propias IES menos alternativas para contratar docentes, siendo resultado de las políticas de austeridad iniciadas por la crisis económica que amenazó al país desde 2007.

EI Programa ACADEMIA, se avoca a evaluar al docente, pero bajo la expectativa de una contratación permanente bajo las figuras de Profesor Titular de Universidad y Catedrático de Universidad (figura similar al de PTC en México). Sus resultados dan cuenta de una elevada exigencia en los procesos de evaluación, y con la entrada en vigor en noviembre de 2017 de los nuevos requisitos, se alerta del endurecimiento en la mayor parte de las áreas, especialmente, en la relacionada con la investigación docente, provocando dejar a centenares de profesores universitarios sin ascenso.

Finalmente, el Programa DOCENCIA ha propuesto sistemas de evaluación a todo el profesorado mediante asesorías expertas y técnicas y procesos de evaluación internos de cada IES. Sin embargo, la limitación del Programa son los escasos recursos económicos con los que cuentan las IES.

Con respecto a los procesos de evaluación para la mejorar de la calidad de las IES, la ANECA puso en marcha los programas AUDIT, VERIFICA, MONITOR, ACREDITA y ACREDITA PLUS. 
EI Programa AUDIT trata de favorecer y promover el desarrollo e implantación de Sistemas de Garantía Interna de Calidad en las IES, y poner en práctica procedimientos conducentes al reconocimiento y certificación de dichos sistemas, toma como objeto de evaluación la propia gestión administrativa, la formación de los docentes, los planes de estudio, etc. Sus resultados, han otorgado a 26 universidades esta certificación, que sirve para mejorar procesos de mejora de la enseñanza y para rendir cuentas a la sociedad.

EI Programa VERIFICA dirigido a evaluar los planes de estudio (títulos de grado, máster y doctorado oficiales) y títulos oficiales de ES no universitaria, o supervisar las competencias que se adquirirán en la titulación, se ha observado un incremento del número de nuevos títulos oficiales a evaluar, superando el millar de propuestas. Sus resultados confirman una valoración notablemente alta, aunque esta realidad no asegura una pertinencia entre el nivel de ajuste entre la oferta y la demanda por parte del alumnado.

EI Programa MONITOR encargado de evaluar la calidad de los títulos oficiales universitarios, donde contrasta las tasas de rendimiento, abandono o deserción escolar, etc., y comprueba su correcta implantación. Entre sus mejoras ha incluido la definición del plan de estudios con base en un modelo educativo centrado en el estudiante y los propósitos del EEES, repercutiendo en una mayor dedicación del docente y reflexión sobre los resultados que deben lograr los estudiantes, suponiendo una transformación significativa que aún está en proceso.

Mientras el Programa ACREDITA dedicado a la renovación de los títulos de grado, master y doctorado, sus resultados indican una valoración favorable en una alta tasa de títulos de grado y de máster, reflejando el trabajo previamente realizado por las instituciones. El programa ACREDITA PLUS, tiene la particularidad de proporcionar Sellos Europeos de Calidad para los títulos universitarios como reconocimiento de su orientación profesional de sectores científico-técnicos y profesionales en el ámbito europeo, ofreciendo un respaldo a los egresados al incluirlo como mérito en los títulos de Ingeniería (EUR-ACE) e Informática (EURO-INF). Sus resultados arrojan, que del sello EUR$A C E \circledast$, fueron evaluadas positivamente 17 propuestas y del sello EURO-INF 6 propuestas, dando a entender el plus con el que cuentan estas titulaciones y los centros acreditados, repercutiendo en esfuerzos de otras titulaciones con las que compensar esta desventaja hacia los egresados (p.e., prácticas en empresas, etc.).

Finalmente, la Comisión Nacional de Evaluación de la Investigación (CNEAI), responsable de la evaluación de la actividad investigadora y de otorgar los tramos de investigación (sexenios), ofrecen al docente un elemento de orientación y reconocimiento para la mejora en su labor a través de diversos incentivos (económicos, incremento o no de carga docente, etc.). Aunque, desde 2012 introdujo el concepto de sexenio vivo, que consistía en que aquel docente que no contase con un tramo de investigación activo, podría ver incrementada su carga docente hasta un máximo de 32 créditos ECTS, perdiendo así el rol histórico de incentivo positivo de investigación.

\section{DISCUSIÓN Y CONCLUSIONES}

La comparación entre los sistemas de evaluación de la ES entre México y España hace evidente sus diferencias y similitudes para diseñar e implementar políticas y programas que mejoren la calidad de la ES.

Entre sus diferencias, México cuenta con una historia y cultura de evaluación de la ES larga, mientras que en España es reciente. Además, el sistema de ES mexicano es amplio y diverso, originando un conjunto de agencias de evaluación gubernamentales y no gubernamentales sumados a un conjunto de programas viejos y nuevos de evaluación y acreditación. Mientras que el español es más sencillo, dado que respondió a una menor heterogeneidad y volumen de instituciones, y centralizado en el gobierno.

Sobre las similitudes ambos países, han creado políticas y programas de evaluación, acreditación y certificación, con el propósito de regular y mejorar la calidad de la ES y rendir cuentas a la sociedad.

México ha conseguido avances en los impactos y efectos de los programas de evaluación, mientras que España, los resultados de sus programas de evaluación son un tema pendiente, aunque entre 
sus buenas prácticas, se incluye el ajuste normativo y legal al sistema de evaluación unificado, al contrario del caso de México cuyo marco legal de ES es de 1978.

En lecciones por aprender, México no tiene un referente internacional, pero podría considerar la experiencia de integrarse a un bloque educativo regional de mejor calidad, o considerar los rankings internacionales a nivel regional. Otra lección es el Programa AUDIT español que certifica los títulos universitarios, algo que se intentó con el CENEVAL, pero fue duramente criticado y rechazado por las universidades públicas. Y en la evaluación del conjunto de profesores e instituciones como norma 0 reglamento, en este caso, España evalúa de la misma manera a docentes e instituciones, mientras que México sólo se ha encargado de evaluar al sector educativo público, dejando en el sector privado una acción voluntaria.

El gran pendiente para México reside en la actualización legislativa para la ES donde sea obligatoria la rendición de cuentas mediante la evaluación externa y la acreditación de las funciones sustantivas y adjetivas de las instituciones y que los Registros de Validez Oficial de Estudios que se otorgan a los centros privados tengan vigencia determinada, siendo renovables con base a criterios de acreditación. Para España queda pendiente unificar los sistemas de ES con Europa, establecer criterios de sistematización unificados y clarificados de los indicadores existentes, y la creación de un ente específico para la gestión de la evaluación de las universidades que se encargara del seguimiento del proceso.

\section{REFERENCIAS BIBLIOGRÁFICAS}

Agencia Nacional de Evaluación de la Calidad y Acreditación (2016). Informe sobre el estado de la evaluación externa de la calidad en las universidades españolas. Madrid: ANECA.

Albatch, P. (2001). Educación Superior Comparada. El conocimiento, la Universidad y el Desarrollo. Buenos Aires: Universidad de Palermo.

Barrenetxea, M., Cardona, A., y Echebarría, C. (2005). La evaluación externa de la calidad en la educación superior: Reino Unido, Suecia y España. Revista Española de Control Externo, 7(20), 89-118.

Buendía, A. (2011). Evaluación y acreditación de programas en México. Más allá de los juegos discursivos. Diálogos sobre Educación, 2(3), 1-19.

Cox, C. (2013). Políticas de Educación Superior: Categorías para su análisis. En Calderón, J., Teoría y desarrollo de la investigación en educación comparada (pp. 259-285). México, DF: Plaza y Valdés, S.A.

European Commission/EACEA/Eurydice (2015). The European Higher Education Area in 2015: Bologna Process Implementation Report. Luxembourg: Publications Office of the European Union.

González, I. (2006). Dimensiones de evaluación de la calidad universitaria en el Espacio Europeo de Educación Superior. Revista Electrónica de Investigación Psicoducativa, 4(10), 445-468.

Haug, G. (2003): Quality Assurance/Accreditation in the Emerging European Higher Education Area: a possible scenario for the future. European Journal of Education, 38(3), 229-239. doi: 10.1111/1467-3435.00143.

Mendoza, J. (Octubre 2003). La Evaluación y acreditación de la educación superior mexicana: las experiencias de una década. Comunicación presentada al VIII Congreso Internacional del CLAD sobre la Reforma del Estado y de la Administración Pública, Panamá. Recuperado de http://unpan1.un.org/intradoc/groups/public/documents/CLAD/ clad0048003.pdf.

Palomares, D., García, A., y Castro, E. (2008). Evaluación de las instituciones de educación superior: revisión bibliográfica de sistema de indicadores. Revista española de documentación científica, 31(2), 205-229. doi: http://dx.doi. org/10.3989/redc.2008.v31.i2.425.

Rubio, J. (2007). La Evaluación y acreditación de la educación superior en México: un largo camino aún por recorrer. Reencuentro, Análisis de Problemas Universitarios, 50, 35-44. Recuperado de http://www.redalyc.org/articulo. oa? id=34005006.

Schriewer, J. (1997). Sistema Mundial y redes de interrelación: la internacionalización de la educación y el papel de la investigación comparada. Educación y Ciencia. Nueva época, 1(1), 21-58. 